A critical re-examination on conduction processes in gasinsulated DC devices at low electric fields

Journal Article

Author(s):

Tschentscher, Malte (1); Franck, Christian (1)

Publication date:

2018-08

Permanent link:

https://doi.org/10.3929/ethz-b-000231185

Rights / license:

In Copyright - Non-Commercial Use Permitted

Originally published in:

IEEE Transactions on Dielectrics and Electrical Insulation 25(4), https://doi.org/10.1109/TDEI.2018.007259 


\title{
A Critical Re-examination on Conduction Processes in Gas-Insulated DC Devices at Low Electric Fields
}

\author{
Malte Tschentscher and Christian M. Franck \\ High Voltage Laboratory, ETH Zurich, 8092 Zurich, Switzerland
}

\begin{abstract}
The established theories behind low-field ion-drift currents assume that the charge generation from natural ionization is the main source of the charge-carriers, and that it defines the current flowing through the insulation gas in gas-insulated direct current (DC) devices. This charge generation is assumed to be independent of the applied electric field in the cases where the electric field is sufficiently below the onset of microdischarges at the interfaces. However, the results of a few previous studies have suggested the presence of a field-dependent current contribution from some conduction processes, which might significantly accelerate the decay of the surface charges distributed on the insulator surfaces. In this paper, an extensive study on low-field charge transport is presented, and the influences of the insulation volume, electric field, and gas pressure on low-field charge transport are discussed. In accordance with the common structure employed in gas-insulated devices, $\mathrm{Al}_{2} \mathrm{O}_{3}$-filled epoxy resin is used in the solid parts of the device and sulfur hexafluoride $\left.\mathrm{SF}_{6}\right)$ is used for gaseous insulation. At electric fields of approximately $1800 \mathrm{~V} / \mathrm{m}$, a gas current that is up to 30 times higher, when compared to the conduction currents caused by the charge generation from natural ionization, is observed. This enhanced current reveals a further conduction process acting in a limited range of low electric fields and is characterized to be independent of the applied voltage polarity. With an increase in the gas pressure, the current amplitude is found to increase linearly and higher electric field is required to reach its maximum. At electric fields below $200 \mathrm{~V} / \mathrm{m}$, the conduction current reverses its pressure dependency and increases with decreasing gas pressure. The reported findings strongly indicate that the enhanced low-field charge transport in gasinsulated devices may be owing to electrophoretic conduction.
\end{abstract}

Index Terms - Charge generation, surface charge, charge decay, electrostatic probe, epoxy resin, $\mathrm{SF}_{6}$, natural ionization, GIS, DC, ion current, electrophoresis

\section{INTRODUCTION}

A detailed understanding of the low-field charge generation and transport in gaseous insulation forms the basis for modeling numerous problems in high-voltage engineering. Some examples for processes present in gas-insulated direct current (DC) devices are surface-charging and charge decay phenomena at insulator surfaces, the prediction of initiatory electron provisions for partial discharges in small voids [1], the estimation of statistical time lags for breakdown inception in gaseous insulation $[2,3,4]$, etc. Here, the intensity and continuity of the low-field charge provision strongly influence the operating states of the devices and, owing to their impact on the mean energy input from the discharges [5], the longterm performance and aging behavior of the insulation.

The established theories of charge generation and transport under low electric fields have been mainly obtained from

Manuscript received on 10 December 2017, in final form 5 March 2018, accepted 5 March 2018. Corresponding author: M. Tschentscher. continuous ion-current measurements $[2,16]$. The conduction currents through a gas gap were measured for different electric fields, gas pressures, and insulation volumes $[2,11,16]$. For the analyzed fields, which were sufficiently below the onset of microdischarges at the interfaces, the charge generation was primarily assumed to correspond to ion pair (IP) generation resulting from natural ionization in the gas. Throughout the literature, this assumption was applied for the simulation of surface-charge accumulation on solid insulators installed in HVDC devices, and therefore, for the dimensioning of solid-gas insulation systems [7-10]. Under the conditions described, the IP rates were mostly considered to be independent of the gas volume and electric field. In contradiction to this assumption, it has recently been shown that the surrounding solid materials may also influence the charge provisioning in the insulation gas significantly [11]. In particular for small insulation distances, the IP rate was found to be strongly dependent on the gas volume. While this dependency explained the charging phenomena arising from saturated ion currents under electric fields above $5000 \mathrm{~V} / \mathrm{m}$, 
discharging experiments with locally limited surface-charge distributions formed from microdischarges indicated the presence of further low-field conduction processes that could change with the electric field amplitude [11]. The discharging of such locally limited surface-charge patterns could not be explained quantitatively, using the charge generation from natural ionization and the common ion current models. A few authors who investigated the surface-charge accumulation on model spacers downscaled from gas-insulated components also observed similar discrepancies [12-15]. Some authors attributed the increased low-field charge transport to electrophoretic conduction caused by dust particles in the gas $[12,13,15]$. A few others suggested that an enhanced level of humidity could considerably increase the charge transport in the gas $[16,17]$.

It should be noted that experimental studies on full-size HVDC equipment and scientific arrangements have indicated that the surface-charge accumulation under technical conditions is commonly dominated by microdischarges at interfaces [18,19] and thermal effects in the solids [20,21]. This contradicts the relevance of low-field conduction phenomena for the dimensioning of gas-insulated devices. However, low-field conduction phenomena influence the surface charge decay at no-load conditions and might be of interest for the planning of test procedures. One example is the determination of reasonable waiting times during polarity reversal tests.

This study focuses on the charge transport under low electric fields and dry conditions, which are far below the inception of microdischarges at the interfaces and at humidity below $200 \mathrm{ppm}_{\mathrm{v}}$. Conduction currents in the sub-fA range were obtained from the surface-charging experiments, by calculating the time derivatives of the surface-charge distributions measured at the epoxy-gas interfaces. To address the scalability of the low-field conduction phenomena, experiments were performed with different insulation volumes, electric fields, and gas pressures.

\section{EXPERIMENTAL SETUP}

Figure 1 shows the setup developed to scan the twodimensional (2D) surface potential distributions of polymeric plate samples charged with an HVDC electrode. The scan system was placed inside a sealed chamber designed for gas pressures up to $0.6 \mathrm{MPa}$. The atmospheric conditions within the chamber, namely, the humidity, gas pressure, and temperature, were monitored continuously using a dew point meter (MBW 973) and a combined capacitive sensor (WIKA GDHT-20). The surface potentials at the polymer-gas interface were obtained through contactless measurements using an electrostatic probe (Trek 341B). High-voltage (HV) potentials were supplied by a $60 \mathrm{kV}$ DC source (Heinzinger PNC hp 60000-3 mA) monitored with a 16-bit digital interface.

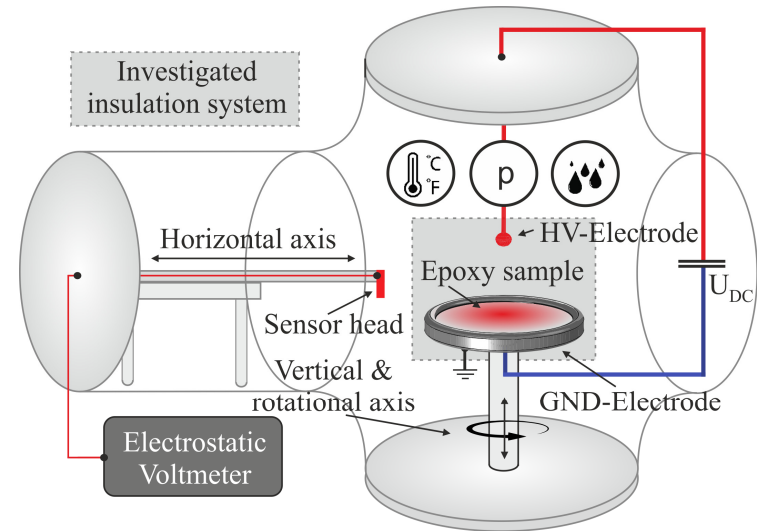

Figure 1. Schematic diagram of experimental setup (adapted from [11]).

\subsection{INVESTIGATED INSULATION SYSTEM}

The insulation system consisted of a $4 \mathrm{~mm}$ thick epoxy plate, which was filled with aluminum oxide $\left(\mathrm{Al}_{2} \mathrm{O}_{3}\right)$ microparticles, and a gas volume of sulfur hexafluoride $\left(\mathrm{SF}_{6}\right)$. The epoxy plate was embedded on to a grounded (GND) aluminum electrode and the gaseous domain was bounded at the upper end by a spherical HV electrode of radius $7.5 \mathrm{~mm}$. Therefore, the electric field lines spanning from HV to GND defined the investigated insulation system. The distance between the HV electrode and the sample surface determines the gas volume during surface-charging experiments, which contributed to the surface-charge accumulation on the investigated surface area of the polymer-gas interface, hereafter called the active gas volume. The surface potentials were measured for a $0.25 \times 0.25 \mathrm{~mm}^{2}$ scan raster, with the sensor placed at a distance of $2 \mathrm{~mm}$ from the sample surface, which resulted in a tradeoff between the overlap of the sensor response functions (SRF) and the signal amplitude. The SRF describes the influence of the surface charges on the sensor potential at various distances from the sensor axis.

In accordance with the data processing introduced in [11], an inverse model was used to calculate the charge distributions from the surface potential scans. By deconvoluting the two-dimensional potential scans using the $\mathrm{SRF}$, the charges were computed considering the measurement principle of the electrostatic probe, which minimizes the electric field centrally below the sensor head. The SRF was found to be independent of the sensor position for sample surface radii lesser than $50 \mathrm{~mm}$ from the sample center. In this study, the charge distributions were computed for a resolution of $0.1 \times 0.1 \mathrm{~mm}^{2}$. More detailed descriptions of the measurement principle, experimental setup, and numerical model can be obtained from [11]. 


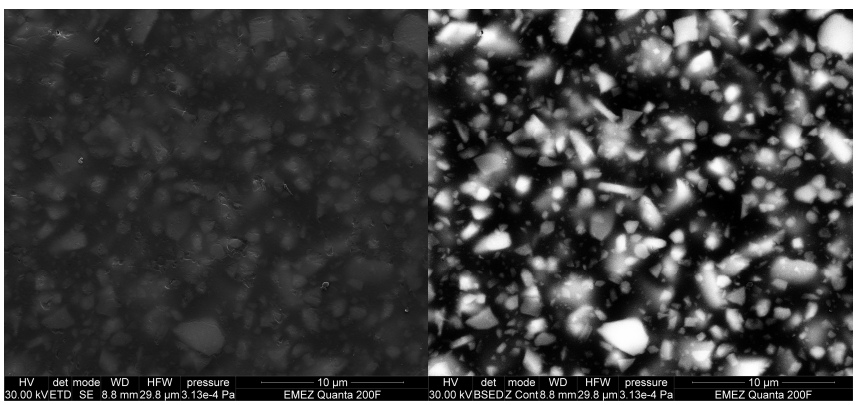

Figure 2. Scanning electron microscope (SEM) picture of $\mathrm{Al}_{2} \mathrm{O}_{3}$-filled epoxy plate samples (left: secondary electron (SE) signal, right: backscattering electron (BSE) image).

\subsection{SAMPLE PREPARATION}

Prior to the experiments, the vertically casted epoxy samples were pre-dried and degassed under vacuum at $80^{\circ} \mathrm{C}$ for at least three days. Figure 2 shows the scanning electron microscope (SEM) images from the sample center. The secondary electron (SE) signal showed a smooth surface structure covered by a thin epoxy film that was only sporadically punctuated by filler particles. The backscattering electron (BSE) image highlighted the filler particles, owing to their higher effective nuclear charges, showing a homogenous and un-agglomerated filler distribution. Similar surface compositions were observed on the disc spacers of the gasinsulated switchgears also.

\subsection{PRECISION OF CHARGE CALCULATIONS}

The accuracy of the developed scan system could be derived from the measurements obtained using a calibration electrode as shown in Figure 3. The grounded electrode included six insulated needles with tip radii of $0.5 \mathrm{~mm}$, which lined up precisely with the electrode surface. To validate the shape of the theoretically obtained SRF, a voltage was applied to the needles and the electrode potentials were scanned. The shape of the measured SRF, agreed very well with the simulated one obtained with finite-element methods. A quantitative comparison was not possible as the used electrode system during calibration differed from that of the investigated insulation system introduced in Section 2.1. The standard deviation of the sensor positioning was found to be $0.01 \mathrm{~mm}$ per $\mathrm{mm}$ increment.
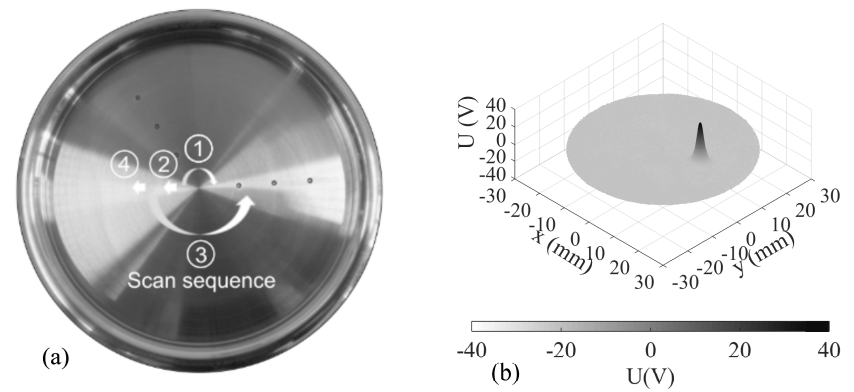

Figure 3. (a) Electrode used to calibrate the scanning system, (b) plot of 2D surface potential scan when applying voltage to one of the needles, with a scan resolution of $0.25 \times 0.25 \mathrm{~mm}^{2}$.

A discharge experiment was performed to determine the measurement limits when deriving the conduction currents from the time derivatives of the surface charges. During the discharge, the surface potentials of an epoxy sample were measured repeatedly using the system configuration described in Section 2.1. The HV electrode was placed at a distance of $100 \mathrm{~mm}$ from the sample surface and was grounded throughout the experiment. In this manner, the reproducibility and fluctuations of the surface charge calculations were analyzed. The surface potentials were scanned hourly and the corresponding surface charge densities were derived by deconvoluting the 2D surface potential scans with the SRF. Figure 4a shows the time series of the surface charges, which is obtained by integrating the surface charge densities over a circular area of $12 \mathrm{~mm}$ radius from the sample center. After a discharging period of $30 \mathrm{~h}$, the charge series flattened with respect to time. Therefore, the remaining $50 \mathrm{~h}$ were used to determine the noise and drift of this measurement technique. Assuming the worst case of charge fluctuation, by considering the difference between the maximum and minimum charges in this period and assuming the difference to be charged within one hour, a maximal noise amplitude of $0.14 \mathrm{fA}$ was obtained. However, for discussing experiments in this paper, three-hour charging currents were derived by fitting the charge derivatives of these time intervals with a linear regression model. Figure $4 \mathrm{~b}$ shows the corresponding three-hour charging currents obtained for measurement times larger $30 \mathrm{~h}$. It was found that $90 \%$ of the currents, framed by the $95 \%$ and $5 \%$ quantiles, were measured in the range from -10 to $+11 \mathrm{aA}$, and therefore, were even one order of magnitude lower than the previously derived noise maximum. Moreover, the mean current was close to $0 \mathrm{~A}$ and no significant current drift was observed.
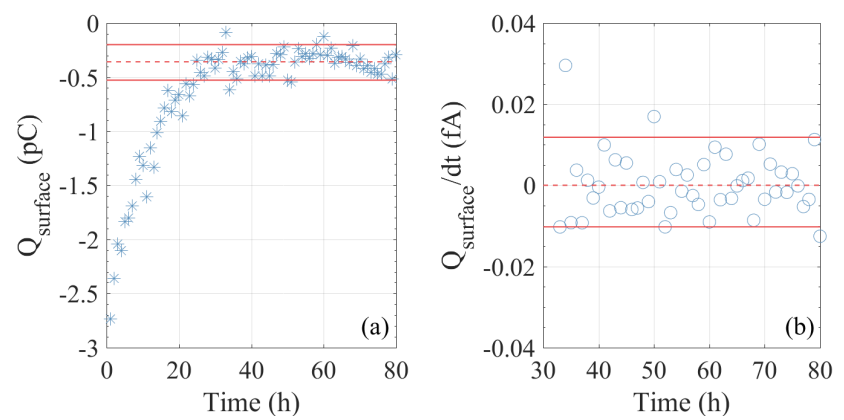

Figure 4. (a) Time series of surface charges and (b) three-hour charging currents for measurement times larger $30 \mathrm{~h} .90 \%$ of the values lie between the red lines, and the mean values for times longer than $30 \mathrm{~h}$ are shown in dashed red.

\section{EXPERIMENTAL RESULTS}

The focus of this contribution is on the surface-charge accumulation at the epoxy- $\mathrm{SF}_{6}$ interface, which is caused by the conduction and charge generation in the gas. Three series of measurements were conducted, each of which included numerous experiments with the same epoxy sample and HV electrode. Thus, the influences of gas volume, electric field, and gas pressure on the low-field conduction currents though $\mathrm{SF}_{6}$ were analyzed. Each experiment produced a measured time series of surface potential scans taken either hourly or two-hourly. For each potential scan, the surface-charge distribution was calculated using the numerical model 
described in Section 2.1. Figure 5 shows the time series of surface-charge accumulation obtained from surface potential scans. The charging currents, contributing to the charging of the sample surface, were calculated by fitting the three-hour time derivatives of the charge series to a linear regression model. The currents were investigated for four distances (40, 90,200 , and $300 \mathrm{~mm}$ ) between the spherical $\mathrm{HV}$ electrode and the sample surface. For these distances, the electric field lines defined the corresponding gas volumes to be $11,25,58$, and $87 \mathrm{~cm}^{3}$, respectively. All experiments were conducted at temperatures of $22{ }^{\circ} \mathrm{C} \pm 1{ }^{\circ} \mathrm{C}$ and humidity below $200 \mathrm{ppm}_{\mathrm{v}}$, which were stabilized by the use of a desiccant.

It should be noted that a few low-field experiments measured with an electrode distance of $40 \mathrm{~mm}$ and gas pressure of $0.4 \mathrm{MPa}$ were performed with a spherical electrode that was additionally tipped with steel in a $10 \mu \mathrm{m}$ radius. Similar to the electrode used in [11], the microtip was placed in line with the rotation axis of the sample, oriented towards the sample surface. For this configuration and conditions, the onset voltage for microdischarges was previously found to be approximately $3 \mathrm{kV}$ [11], which corresponded to an electric field of $3 \times 10^{4} \mathrm{~V} / \mathrm{m}$ at the gas side of the sample surface. Because the discussed experiments, conducted with the tipped electrode, were measured under far lower fields, not showing any contribution from microdischarges their results are not separately mentioned in the following discussions. This approach was validated by the measurements conducted with an untipped electrode under the same conditions, which showed similar charging currents.
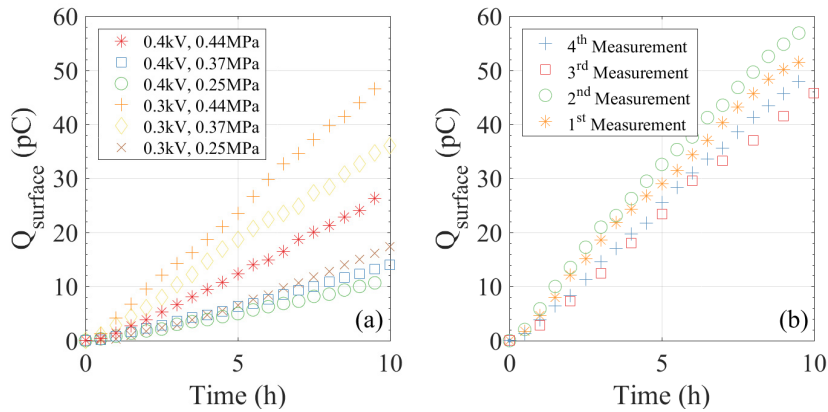

Figure 5. (a) Surface charges as functions of the charging time at different gas pressures and voltages, measured when placing the electrode at a distance of $40 \mathrm{~mm}$, (b) fluctuations in surface charging when applying a voltage of $0.2 \mathrm{kV}$ at $0.44 \mathrm{MPa}$ and placing the electrode at a distance of $40 \mathrm{~mm}$.

Figure 5 shows the time series of the first low-field charging experiments conducted with an electrode distance of $40 \mathrm{~mm}$ and voltages of $0.2 \mathrm{kV}, 0.3 \mathrm{kV}$, and $0.4 \mathrm{kV}$. For all experiments, the surface charges increased to a lesser extent within the first measurement hour compared to the rest of the time series. This behavior agreed with those of the experiments presented in [11] and could be directly attributed to the charging currents from the bulk, which contributed with the opposite current polarity, in respect to the charging currents from the gas, to surface charge accumulation at the sample surface. It most probably resulted from the polarization processes that showed a time-limited response, and did not contribute to the charging currents after the polarization became stable. As this contribution focused on charge generation and transport in the gas, the first hour of the charging experiments was neglected in the calculation of conduction currents from the slope of the surface charging time-series. Here, charge accumulations were found to follow a continuously rising trend over time, which was linearly dependent on the applied voltage, and showed a decrease in the slope with an increase in the voltage. No significant shortterm fluctuations of the charging slope were observed during each $10 \mathrm{~h}$ experiment. However, experiments performed sequentially at $0.2 \mathrm{kV}$ over two weeks revealed current fluctuations of up to $30 \%$ in amplitude without any clear tendency toward higher or lower values. Nevertheless, from the continuous and linear charge rise within each experiment, it could be concluded that the influence of the surface charges on the electric fields, and therefore, on the charge generation and transport, were negligible.

A pressure dependency was observed for experiments conducted at $0.3 \mathrm{kV}$ and $0.4 \mathrm{kV}$, plus a dependency on the applied voltage. The currents increased disproportionately with the gas pressures, by factors of approximately 1.42 and 1.68 per $0.1 \mathrm{MPa}$ for voltages of $0.3 \mathrm{kV}$ and $0.4 \mathrm{kV}$, respectively. However, the limited data basis of the first measurement series, considering the current fluctuations mentioned before, did not allow to draw a clear conclusion regarding the pressure dependencies of the low-field conduction phenomena.

Figure 6 shows the charge distributions obtained from the surface-charging experiments conducted with the HV electrode at a distance of $40 \mathrm{~mm}$ under an applied voltage of $0.2 \mathrm{kV}$, which produced the highest charging currents. Flat charge plateaus could be observed with slightly accentuated microstructures. In accordance with the previously published saturated charging currents [11], the microstructure of the charge distribution became more pronounced as the charging time increased. The positive surface-charge accumulation for a positive applied voltage could be directly attributed to the domination of the gas domain over the conduction and polarization in the solid insulation [11]. Comparable charge patterns could be measured, differing primarily in their rise times, over the entire voltage range under consideration. On comparing these measurement series with the experiments conducted at higher electric fields and those published in [11], a significant increase in the charge accumulation was observed at low electric fields.

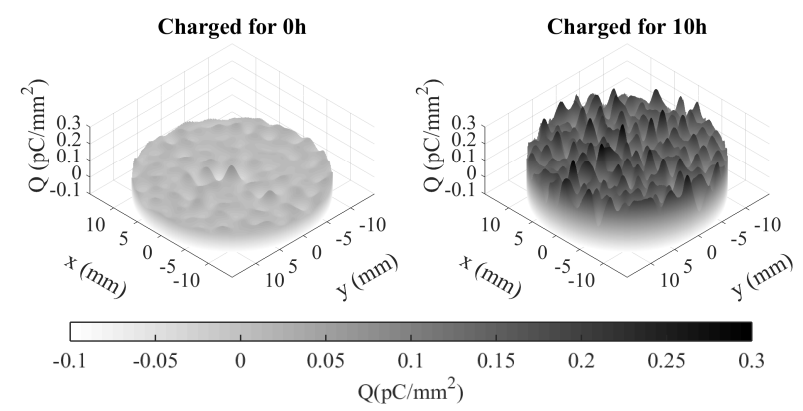

Figure 6. Surface charge densities obtained when placing the electrode in $40 \mathrm{~mm}$ distance and applying a voltage of $0.2 \mathrm{kV}$.

To understand this phenomenon better, a second measurement series that analyzed the field and volume 
dependency of this enhanced low-field conduction was generated for gas pressures in the range of $0.4 \mathrm{MPa}$ to $0.45 \mathrm{MPa}$. The surface charging time series were compared in terms of the averaged normal electrostatic fields $\left(E_{\text {avg,gas }}\right)$ simulated for the gas side of the epoxy-SF 6 interface. Supported by the measurements presented in Figure 5, the influences of the surface charges and space charges in the gas on the electric fields were neglected for the chosen parameters. The sample remained unchanged within this measurement series even though gas handling was performed several times during the experiments; however, this did not influence the measurement results. Figure 7 depicts the charging currents calculated for different electrode distances, in terms of the applied voltages and the corresponding electrostatic fields at the gas side of the interface ( $\left.E_{\text {avg,gas }}\right)$. In order to compare the charging currents from the experiments with positive and negative voltages applied to the HV electrode in the gas, the currents were multiplied with the sign of the applied voltage, which was described mathematically by $U /|U|$. Thus, the charging currents originating from the gas contributed positively to the change in the surface charge multiplied with the voltage sign, and the reverse was true for a dominant conduction through the solid sample.

The experiments revealed a peak-like current dependency on the applied voltage. The measured current peaks scaled with the voltage and shifted for an increasing electrode distances to higher voltage values. Whereas the peak shifts with the voltage, these conduction phenomena correlated with the averaged electrostatic field at the gas side of the epoxy$\mathrm{SF}_{6}$ interface. A high reproducibility of the currents was demonstrated when alternating the electrode distances and applied voltages during the measurement series, without observing a dependency on their succession. The currents shown for $E_{\text {avg,gas }}$ beyond $5 \times 10^{3} \mathrm{~V} / \mathrm{m}$ and the corresponding voltages were taken from the charging experiments presented in [11].

We start the analysis of Figure 7 from zero applied voltage. First, the current increased steeply with an increase in the electric field, until it reached its maximum at an electric field of approximately $1800 \mathrm{~V} / \mathrm{m}$. The rise was followed by a decrease in the current, before it flattened for a field of approximately $4000 \mathrm{~V} / \mathrm{m}$. Beyond this limit, the current showed a weak field dependency, which was previously explained to be caused by the generation of saturated ion currents $[2,11]$ from the natural ionization in the gas and ohmic conduction through the solid insulation [11]. When compared to the saturated conduction regime, only the currents at fields below $100 \mathrm{~V} / \mathrm{m}$ showed similar amplitudes. A significant deviation from the saturated conduction currents was observed for the peak values, which were measured to be up to 30 times higher. Here, in contrast to the increase in the saturated currents towards larger gas volumes, the low-field peaks did not show a linear volume scaling. Experiments conducted with electrode distances of $40 \mathrm{~mm}$ and $300 \mathrm{~mm}$ revealed current peaks lower than those observed for distances of $90 \mathrm{~mm}$ and $200 \mathrm{~mm}$. Moreover, no difference in currents was observed in the experiments conducted with different voltage polarities but same amplitudes.
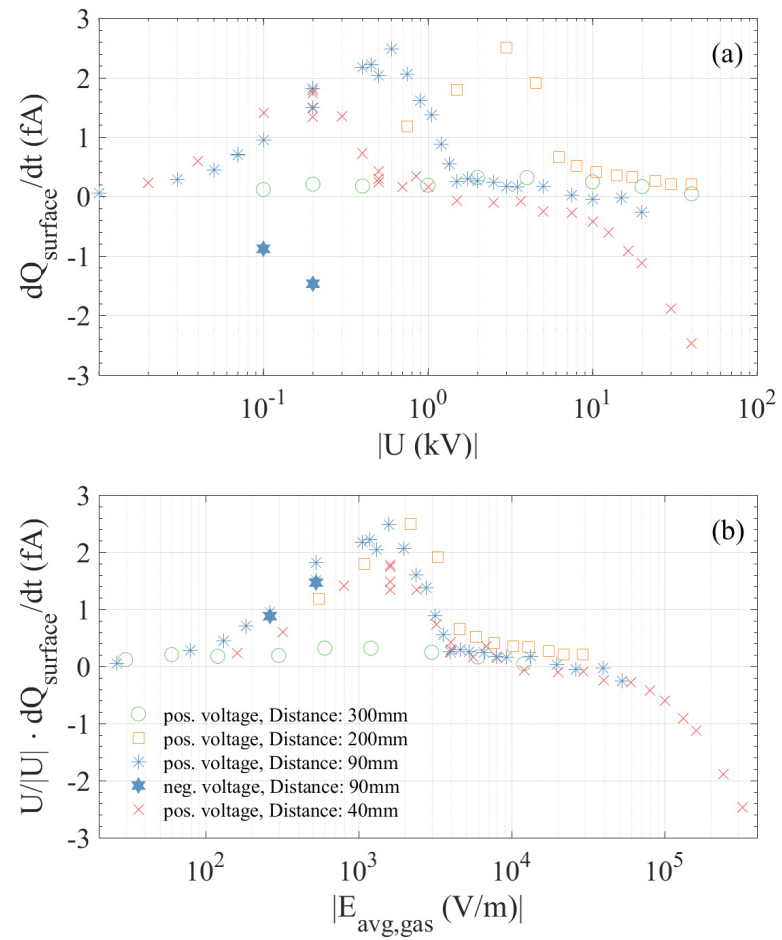

Figure 7. Charging currents for various applied voltages (a) and electrostatic fields $E_{\text {avg,gas }}(\mathrm{b})$ at the gas side of the epoxy-SF 6 interface (values for $E_{\text {avg,gas }}$ larger $5000 \mathrm{~V} / \mathrm{m}$ and the corresponding voltages were taken from [11]).

For analyzing the pressure dependencies of the low-field current peaks and for comparing them with the previous experiments conducted in the pressure range from $0.4 \mathrm{MPa}$ to $0.45 \mathrm{MPa}$, a third series with gas pressures of $0.25 \mathrm{MPa}$ and $0.125 \mathrm{MPa}$ and voltages ranging from $10 \mathrm{~V}$ to $10 \mathrm{kV}$ was produced. Figure 8 shows the charging currents measured while placing the HV electrode at a distance of $40 \mathrm{~mm}$ from the sample surface. With a decrease in the gas pressure, the current peak shifted towards lower fields and decreased in amplitude. Within the flat current regime, no significant changes were observed in the conduction currents.

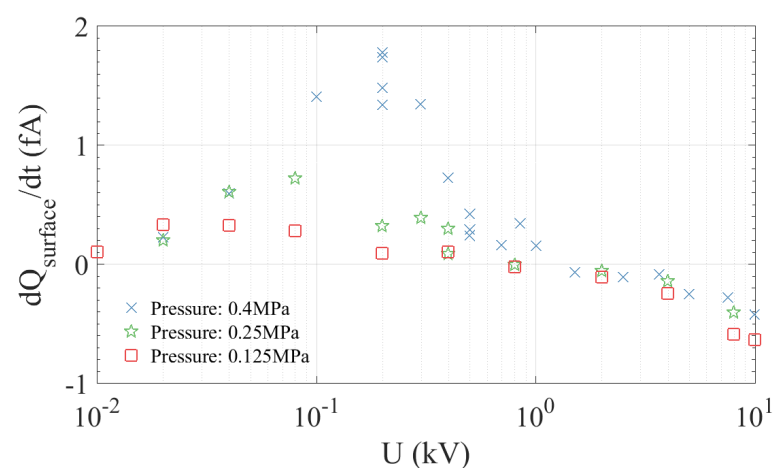

Figure 8. Charging currents for different gas pressures when placing the electrode at a distance of $40 \mathrm{~mm}$ from the sample surface (values for $0.4 \mathrm{MPa}$ gas pressure and voltages larger $1 \mathrm{kV}$ were taken from [11]).

\section{DISCUSSION}

The established understanding of ion-drift currents at low electric fields below the onset of microdischarges assumes 
natural ionization as the only source of charge provision. The amount of IPs generated per unit volume, pressure, and time, depended on the altitude [23], shielding of the device [24], and the properties of the gas, such as the atomic number [23]. In line with this theory, the conduction current through a gas gap was expected to increase linearly with an increase in the gas volume. The saturated currents in the gas were usually assumed to be determined exclusively by the amount of IP generation in the gas from the interaction of cosmic and terrestrial radiation with $\mathrm{SF}_{6}$. However, recently it was shown that the surrounding solids, namely, the metallic electrodes or solid insulators, could also contribute to the charge provision in the insulation gap [11]. The results indicated a contribution of $0.85 I P \mathrm{~cm}^{-3} \mathrm{~s}^{-1} \mathrm{bar}^{-1}\left(I P_{\text {solid }}\right)$ per square millimeter of the solid-gas interface (S) [11]. Taking the conduction currents through the solid insulation $\left(I_{\text {solid }}\right)$ into consideration, the change of surface charges, could be described as:

$$
\begin{aligned}
& \frac{U}{|U|} \cdot \frac{d Q_{\text {sufface }}}{d t}=\underbrace{e \cdot p \cdot V_{\text {gas }} \cdot\left(I P_{\text {gas }}+S \cdot I P_{\text {solid }}\right)}_{I_{\text {gas }}} \\
&-\underbrace{\int \sigma_{V}(T) \cdot E_{\text {avg,solid }} \cdot d A}_{I_{\text {solid }}} .
\end{aligned}
$$

By subtracting $I_{\text {solid, }}$, calculated with a volume conductivity $\left(\sigma_{\mathrm{V}}\right)$ of $0.9810^{-16} \mathrm{~S} / \mathrm{m}$ at $22{ }^{\circ} \mathrm{C}$ [11], from the change in the surface charges, the charging currents from the gas side of the interface could be derived. It should be noted that this derivation assumed homogeneously distributed charge generation as the only source of charge carriers in the gas.

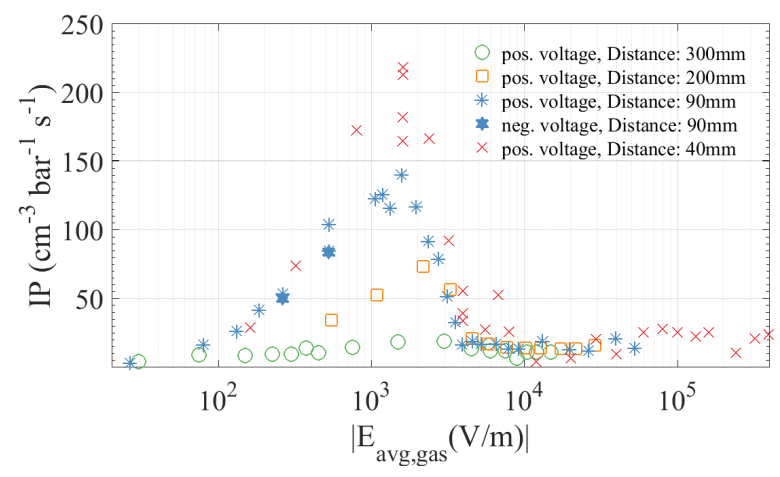

Figure 9. IP rates for different averaged electric fields at the gas side of the sample surface at gas pressures in the range of $0.4 \mathrm{MPa}$ to $0.45 \mathrm{MPa}$ (values for $E_{\text {avg,gas }}$ larger $5000 \mathrm{~V} / \mathrm{m}$ were taken from [11]).

Figure 9 shows the apparent IP rates derived for experiments conducted with gas pressures in the range of $0.4 \mathrm{MPa}$ to $0.45 \mathrm{MPa}$. In line with the observed behavior of conduction currents at low electric fields, but contradictory to the established conventions, a peak-like enhancement was observed in the IP rates. In the range of $100 \mathrm{~V} / \mathrm{m}$ to $5000 \mathrm{~V} / \mathrm{m}$, the calculated rates exceeded the values calculated from the saturated current regime up to 30 times. Moreover, the peaklike shape of the rates for different electric fields could not be explained with the established understanding of low-field charge generation from natural ionization.
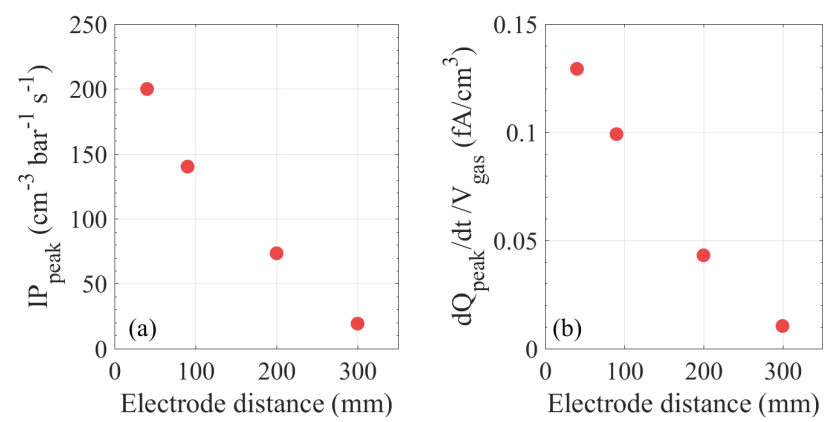

Figure 10. (a) Peak IP rates and (b) peak charging currents per gas volume for different electrode distances, derived from the experiments conducted under gas pressures in the range of $0.4 \mathrm{MPa}$ to $0.45 \mathrm{MPa}$.

Figure 10 shows the maximum derived IP rates (IPpeak) and the corresponding peak currents per gas volume for different electrode distances (d). The values decreased, approximately linearly, with a slope $(\alpha)$ of $4.5 \times 10^{-}$ ${ }^{4} \mathrm{~cm}^{-4} \mathrm{~s}^{-1}$ bar ${ }^{-1}$ at larger distances, which can be described with an additional low-field current term $I_{\text {low-field }}$ In accordance with the established theory for the distributed IP generation per unit volume, pressure, and time, the peak conduction could be described by:

$$
\begin{aligned}
& \frac{U}{|U|} \cdot \frac{d Q_{\text {surface }}\left(E_{\text {peak }}\right)}{d t}=\underbrace{e \cdot p \cdot V_{\text {gas }} \cdot\left(I P_{\text {gas }}+S \cdot I P_{\text {solid }}\right)}_{I_{\text {gas }}} \\
& -\underbrace{\int \sigma_{V}(T) \cdot E_{\text {peak }} \cdot d A \text { feld }}_{I_{\text {solid }}}+\underbrace{e \cdot p \cdot V_{\text {gas }} \cdot\left(I P_{0}-\alpha \cdot d\right)}_{\text {gas }} .
\end{aligned}
$$

Figure 11 shows a comparison of the measured rates with the values obtained from literature, which were derived from continuous ion current measurements conducted with electrode distances larger than $128 \mathrm{~mm}$ and coaxial electrode arrangements, at TU Munich [2]. With an increase in the electrode distance, the low-field peaks of the IP rates became increasingly comparable to the values from TU Munich, which went hand-in-hand with a flattening of the low-field peak. The values for an electrode distance of $300 \mathrm{~mm}$ also showed good agreement with the IP rates calculated from the currents measured in the saturated conduction regime [11].

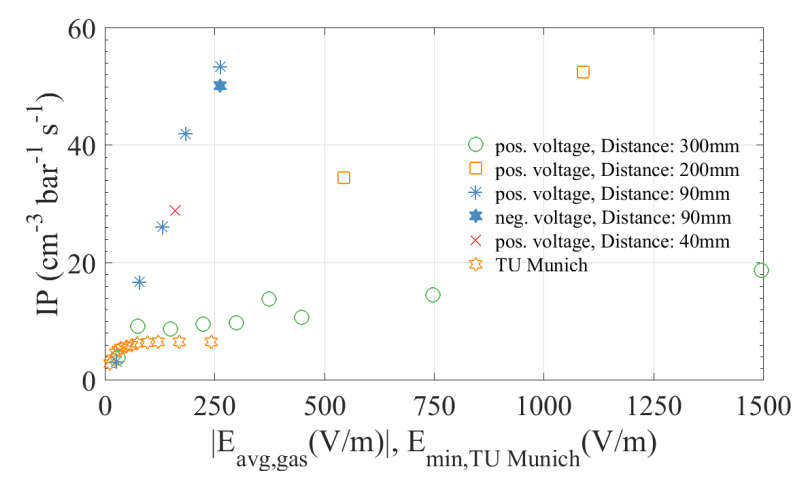

Figure 11. Comparison of low-field IP rates for different $E_{\text {avg,gas }}$ (values for $E_{\text {avg,gas }}$ larger $5000 \mathrm{~V} / \mathrm{m}$ were taken from [11]) with values derived from literature [2] presented for the minimum electric field during these experiments. 

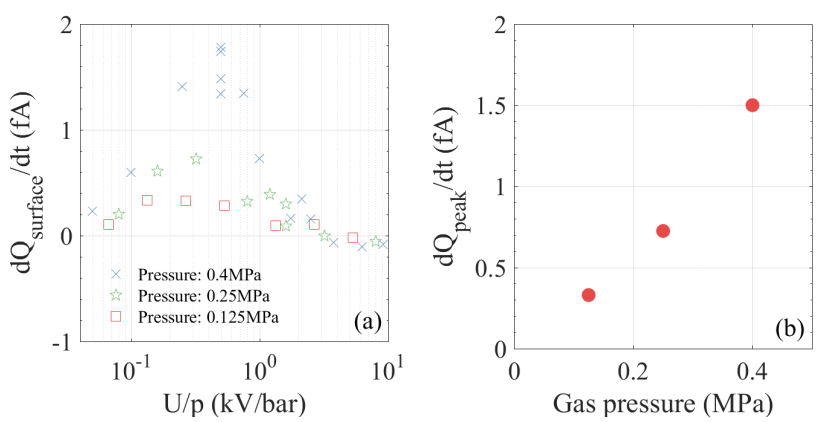

Figure 12. (a) Charging currents for different applied voltages per gas pressure and (b) peak charging currents for different gas pressures for experiments conducted with an electrode distance of $40 \mathrm{~mm}$.

Figure 12a shows the derived charging currents from the third measurement series for various applied voltages divided by the gas pressure. The synchronized peak shape, in comparison with the distribution positions shown in Figure 8, let assume an approximately linear shifting of the peak value with an increase in gas pressure. On comparing the currents in Figure 8 for a fixed low electric field below $200 \mathrm{~V} / \mathrm{m}$, an increase in the current was observed for a decrease in the pressure. This behavior indicated a dependency of the conduction current on the transport parameter, which seem to also define the current amplitude and not only on the charge carrier density as assumed for saturated ion currents. Moreover, a flattening of the peak current was observed for experiments conducted with different gas pressures but the same electrode distance. Figure $12 \mathrm{~b}$ shows an approximately proportional peak current increase with an increase in the gas pressure, which suggests that the current is caused by a charge carrier species that is inserted or extracted via gas handling to the test chamber. While this clear pressure dependency was valid for these experiments, the measurements with a fixed gas pressure but different electrode distances indicate a more complex dependency of the peak current on the electric field, electrode distance, and gas volume.

\subsection{EXPLANATORY APPROACH FOR ENHANCED LOW-FIELD CHARGE TRANSPORT}

In spite of the fact that equation (2) may have been parameterized by the measured peak values, it does not serve as an indication for a physical explanation and does not explain the peak-like shape of the low-field gas currents. Therefore, breaking away from the established understanding of low-field ion-drift currents, which are primarily assumed to be determined by the charge generation from natural ionization, a further conduction mechanism that could reproduce the experimental results is sought.

A few interesting attempts to explain the experimental results focused on electrophoretic conduction, owing to the movement of dust particles, or current contributions from gas impurities, e.g. water molecules. Following some studies that demonstrated the potential contributions of dust particles like aluminum trifluoride $\left(\mathrm{AlF}_{3}\right)$ to the charge transport in $\mathrm{SF}_{6}$-filled insulation systems [15], this charge transport mechanism is discussed in more detail here. $\mathrm{AlF}_{3}$ micro or nanoparticles might be formed in gas-insulated switchgears from partial discharges [5] or disconnector operations [25]. The conclusion that the current contribution is caused by a charge transport mechanism, and not by the charge generation at the interfaces, is supported by the increase in the current with the gas pressure and by its independence from the applied voltage polarity.

The increase in current towards higher fields, forming the rising edge of the low-field peak, can be mainly explained in terms of the increase in the electrostatic force that can enhance the drift velocity of charge carriers and additionally facilitate the movement of larger particles. On the other hand, the decreasing current on the application of a certain field may be caused by the electrostatic adherence of particles at the solidgas interfaces, which lowers the number of charge carriers. The measured pressure dependency of the peak amplitude goes in line with Stoke's law, which describes the frictional force of viscosity on a small sphere moving through a viscous fluid or gas. For a constant particle drift velocity, the balance between the electrostatic and viscous forces can be described by:

$$
\underbrace{q \cdot E}_{F_{\text {electrostatic }}}=\underbrace{6 \cdot \pi \cdot \eta \cdot R \cdot v}_{F_{\text {frictional }}} .
$$

This relationship depicts the dependency of the charge carrier drift velocity $(v)$ in a given electrostatic field $(E)$, as a function of the particle charge $(q)$, particle radius $(R)$, and dynamic viscosity $(\eta)$. The dynamic viscosity tends to increase with an increase in the gas pressure. However, a dependency of these changes on the particle size makes an overall estimation difficult [26]. In simple terms, it can be stated that, an increase in pressure requires larger electric fields to develop the same drift velocity and, assuming the same particle number, to conduct the same current. This relationship agrees with the measured dependency of the peak currents that requires higher electric fields for an increase in pressure. The observed increase in the peak currents with pressure might be attributed to a change in the particle number, considering the particle as an inherent species of the insulation gas. This assumption is also supported by the fact that all measured currents show a good reproducibility even over different measurement series, irrespective of the fact that the experiments were interrupted by several gas handlings where the gas chamber is temporarily evacuated or filled with ambient air. Nevertheless, it is not guaranteed that, for a fixed electrode distance and very low electric fields, the current will increase with the pressure, as expected from the observed dependency for the peak values. An increase in the pressure may increase the friction force in a different amount than the number of charge carriers. In line with this remark, the charging currents are found to increase with a decrease in pressure, for electric fields below $200 \mathrm{~V} / \mathrm{m}$. Nevertheless, the experiments with different electrode distances suggest that the friction force also scales with the electrode distance or gas volume, determining the change in peak current for different electrode distances. In summary, it can be concluded that the observed low field conduction phenomena correspond well to the understanding of electrophoretic conduction. 


\subsection{INFLUENCE OF LOW-FIELD CHARGE TRANSPORT ON SURFACE CHARGE DECAY}

In a previous study [11], the decay of the spatially limited surface charge distributions was discussed. The charge patterns were formed by charging the samples with an overcritical microtip. During the surface charge decay, an enhanced discharge current was observed, which could not be explained in terms of the charge generation from natural ionization and which exceeded the expected current up to 10 times. By comparing the electric fields of these experiments, shown in Figure 13, with the ones discussed within this contribution, it can be concluded that the values are comparable. For the active gas volume, which contributes to the charge decay at the interface, mainly, the fields below $5000 \mathrm{~V} / \mathrm{m}$ are calculated. For this reason, we expect a strong contribution from the observed low-field conduction processes to the discharge current, which may explain the accelerated charge decay.

Figure 13 shows the discharge currents of spatially limited surface charges measured at different gas pressures. In distinction to the experiments presented in [11], the HV electrode is grounded and placed at a distance of $150 \mathrm{~mm}$ from the sample surface. The larger electrode distance and the absence of voltage at the HV electrode, which previously constricted the active gas volume, lower the electric fields in the gas. Moreover, it should be mentioned that also the decrease in surface charges over the discharge time reduces the electric field in the gas. What is interesting to note is that the discharge currents reverse their pressure dependencies at a residual charge of approximately $0.28 \mathrm{nC}$. This behavior is in agreement with the measured increase in charging currents towards lower pressures at small fields, as shown in Figure 8, and it supports the theory that the accelerated charge decay is caused by the low-field conduction processes characterized in this study.
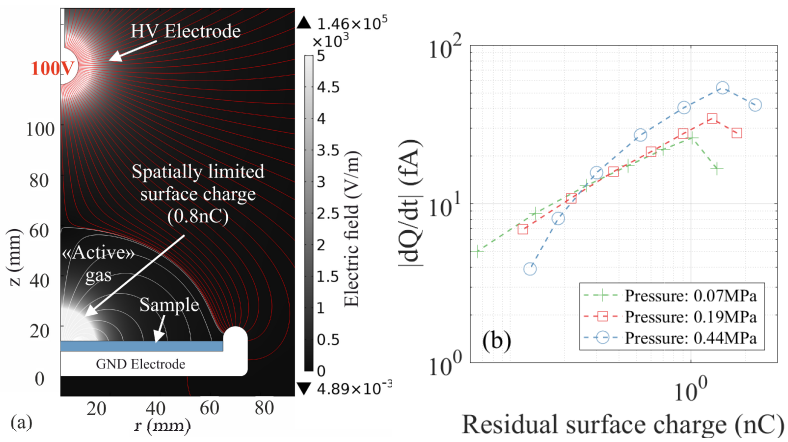

Figure 13. Electric field distributions for discharging experiments with a voltage of $100 \mathrm{~V}$ applied to the HV electrode (a), and the discharge currents for the residual surface charges when grounding the HV electrode (b).

\section{CONCLUSION}

This contribution presented an experimental study on the low-field conduction processes in $\mathrm{SF}_{6}$-insulated devices. A surface-charge measurement setup was used to record the conduction currents in the sub-fA range for different gas pressures, gas volumes, and applied electric fields. From the experiments, the following conclusions were drawn.
- An enhanced charge transport, independent of the voltage polarity, was found for electric fields around $1800 \mathrm{~V} / \mathrm{m}$. The currents, which were up to 30 times higher than the values expected for low-field ion-drift currents, could explain the accelerated decay of localized surface charge distributions observed in previous experiments [11]. The conduction currents could not be reproduced for the charge generation from natural ionization, and vanished for fields higher $10000 \mathrm{~V} / \mathrm{m}$ or for a significant increase in the electrode distance.

- With a change in the electrode distance, while maintaining the gas pressure constant, the observed current amplitudes are a function of the applied voltage, but independent of the electric field. This indicated a correlation between the low-field current contribution and the electrostatic force and supported the hypothesis explaining these behaviors using electrophoretic conduction.

- An increase in gas pressure led to an increase in the current amplitude and shifted the current peak towards higher electric fields. This observation was in line with the expected pressure-dependent increase in gas friction that interfered with the movement of charge carriers. Moreover, the increase in the current amplitude supported the theory that the conduction current was caused by a species dissolved in the gas.

- The low-field current peaks over the gas volume decreased linearly with the electrode distance, which indicated that an increase in electrode distance and therefore gas volume might interfere the low field charge transport processes.

- At very low electric fields below $200 \mathrm{~V} / \mathrm{m}$, the conduction currents increased with a decrease in gas pressure. A similar dependency was observed for the decay of locally limited surface charge distributions.

\section{ACKNOWLEDGMENT}

The authors gratefully acknowledge the technical help and financial support of ABB Switzerland Ltd.

\section{REFERENCES}

[1] F. Gutfleisch, L. Niemeyer; "Measurement and Simulation of PD in Epoxy Voids", IEEE Trans. Dielectr. Electr. Insul. Vol. 2, pp. 729-743, 1995.

[2] J. Kindersberger, "The statistical time lag to discharge inception in $\mathrm{SF}_{6}$ ", Ph.D. thesis, TU München, 1986.

[3] M. Koch, M. Bujotzek, C. M. Franck, "Inception Level of Partial Discharges in $\mathrm{SF}_{6}$ Induced with Short X-Ray Pulses", in Annu. Rep. Conf. on Electr. Insul. Dielectr. Phenom., 2014, pp. 11-14.

[4] N. Wiegart, L. Niemeyer, F. Pinnekamp, W. Boeck, J. Kindersberger, R. Morrow, W. Zaengl, M. Zwicky, I. Gallimberti and S.A. Boggs, "Inhomogeneous Field Breakdown in GIS: The Prediction of Breakdown Probabilities and Voltages", IEEE Trans. Power Del., Vol. 3, pp. 931-938, 1988

[5] C. Beyer, H. Jenett, D. Klockow, "Influence of reactive SFx gases on electrode surfaces after electrical discharges under $\mathrm{SF}_{6}$ atmosphere", IEEE Trans. Dielectr. Electr. Insul. Vol. 7, No. 2, pp. 234-240, 2000.

[6] L. Zavattoni, "Conduction phenomena through gas and insulating solids in HVDC Gas Insulated Substations, and consequences on electric field distribution", Ph.D. thesis, Université de Grenoble, 2015. 
[7] U. Straumann, M. Schüller, C. M. Franck, "Theoretical Investigation of HVDC Disc Spacer Charging in SF6 Gas Insulated Systems", IEEE Trans. Dielect. Electr. Insul. Vol. 19, No. 6, pp. 2040-2048, 2011.

[8] E. Volpov, "Dielectric Strength Coordination and Generalized Spacer Design Rules for HVAC-DC SF 6 Gas Insulated Systems", IEEE Trans. Dielectr. Electr. Insul. Vol. 11, pp. 949-963, 2004.

[9] R. Gremaud, C. B. Doiron, M. Baur, P. Simka, V. Teppati, M. Hering, J. Speck, S. Grossmann, B. Källstrand, K. Johansson, U. Riechert, U. Straumann, "Solid-gas insulation in HVDC gas-insulated systems: measurement, modeling and experimental validation for reliable operation", Cigre Session 49, D1_101, 2016.

[10] M. Tenzer, V. Hinrichsen, A. Winter, J. Kindersberger, D. Imanovic, "Compact Gas-Solid Insulating Systems for High-Field-Stress in HVDC applications”, Cigré Study Committee B3. \& D1 Colloquium Brisbane, paper No. 227, 2013.

[11] M. Tschentscher, C. M. Franck, "Conduction Processes in Gas-Insulated HVDC Equipment: From Saturated Ion Currents to Micro-Discharges", IEEE Trans. Dielectr. Electr. Insul., Issue GI-TL, 2018.

[12] R. E. Wootton, "Electric charge accumulation on HVDC insulators in compressed-SF6-insulated transmission lines", in Proc. IEEE Int. Symp. on Electr. Insul., 1984, pp. 214-217.

[13] A. Bargigia, G. Mazza, A. Pigini, G. Rizzi, "Strength of typical GIS configurations with special reference to composite and combined stresses", Gaseous Dielectrics V, pp. 621-627, 1987.

[14] B. Zhang, Z. Qi, G. Zhang, "Charge accumulation patterns on spacer surface in HVDC gas-insulated system: Dominant uniform charging and random charge speckles", IEEE Trans. Dielectr. Electr. Insul., Vol. 24, No. 2, pp. 1229-1238, 2017.

[15] N. Fujimoto, "Conduction Currents in Gas-Insulated Switchgear for Low Level DC Stress", Gaseous Dielectrics V, pp. 513-519, 1987.

[16] L. Zavattoni, R. Hanna, O. Lesaint, O. Gallot-Lavallée, "Dark current measurements in humid $\mathrm{SF}_{6}$ : influence of electrode roughness, relative humidity and pressure", J. Phys. D: Appl. Phys. 48, 2015.

[17] R. Hanna, O. Lesaint, L. Zavattoni, "Dark Current Measurements in Humid $\mathrm{SF}_{6}$ at High Uniform Electric Field", in Annu. Rep. Conf. Electr. Insul. Dielectr. Phenom., 2016, pp. 19-22.

[18] M. Tschentscher, C. M. Franck, "Microscopic Charge Provision at Interfaces of Gas-Insulated (HVDC/HVAC) Systems", IEEE Trans. Dielectr. Electr. Insul., Issue GI-TL, 2018.

[19] K. Nakanish, "High Voltage DC Gas Insulation" ", Gaseous Dielectrics VI, pp. 433-442, 1991.

[20] C. Y. Li, J. Hu, C. J. Lin, J. L. He, "The Neglected Culprit of DC Surface Flashover-Electron Migration under Temperature Gradients", Scientific Reports, Vol. 7, pp.1-11, 2017.

[21] C. Y. Li, J. Hu, C. J. Lin, B. Y. Zhang, G.X. Zhang, J. L. He, "Surface Charge Migration and dc Surface Flashover of Surface-modified Epoxybased Insulators", J. Phys. D: Appl. Phys., Vol. 49, pp.445304, 2017
[22] M. A. Noras, "Non-contact surface charge/voltage measurements: Capacitive probe principle of operation", Trek Application Note No. 3001, 2002.

[23] C. Patrignani et al. (Particle Data Group), "The Review of Particle Physics 2017”, Chin. Phys. C. 40, 2017.

[24] J. E. Morgan, W. M. Nielsen, "Cosmic-Ray Shower Production and Absorption in Various Materials", Phys. Rev., Vol. 50, 1936.

[25] J. Shea, "High Current AC Break Arc Contact Erosion," in Proc. 54 IEEE Holm Conference on Electrical Contacts, 2008, Orlando, USA.

[26] W. C. Hinds, "Aerosol Technology: Properties, Behavior, and Measurement of Airborne Particles", $2^{\text {nd }}$ Edition, ISBN: 978-0-471-19410-1, 1999.

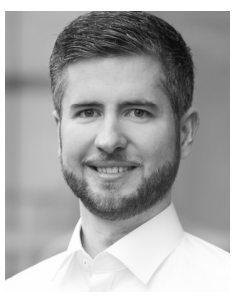

Malte Tschentscher (M17) was born in Recklinghausen, Germany, in 1986. He received the M.Sc. degree in electrical engineering with distinction from RWTH Aachen University, Germany, in 2013. He is currently Scientific Assistant at the High Voltage Laboratory, ETH Zurich, Switzerland. His main research interests include the area of solid and gaseous insulation systems, interface processes and equipment for HVDC.

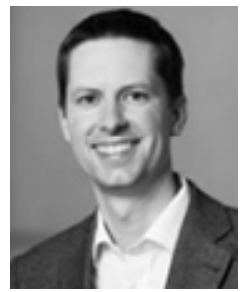

Christian M. Franck (M04-SM11) received a diploma in physics from the University of Kiel, Germany in 1999 and the Ph.D. degree in physics from the University of Greifswald, Germany in 2003. He was with the Swiss corporate research center of ABB during 2003-2009 as a Scientist and Group Leader for gas circuit breakers and high voltage systems. Currently, he is Associate Professor for High Voltage Technology at ETH Zurich, Switzerland. His main research interests are in the area of gaseous insulation systems and equipment for HVDC. 